\title{
A Covid-19 Case Showing Atypical Clinical and Radiological Course with Bilateral Pleural Effusion and Consecutive Negative PCR Test
}

\author{
Sezai Çelik ${ }^{1}$, Nazmi Demirtaş², Nermin Eriz ${ }^{3}$, Yaşar Bükte ${ }^{4}$ \\ Departments of ${ }^{1}$ Thoracic Surgery, ${ }^{2}$ Chest Diseases, ${ }^{3}$ Infectious Diseases, Avicenna Hospital, Istanbul, Turkey; ${ }^{4}$ Department of \\ Radiology, University of Health Sciences Umraniye Training and Research Hospital, Istanbul, Turkey.
}

\section{Corresponding Author:}

Assoc. Prof. Sezai Çelik

Email: siyamie@gmail.com

This is an Open Access article distributed under the terms of the Creative Commons Attribution License (creativecommons.org/ licenses/by/3.0).

Received Accepted

Published

July 13, 2020

November 1, 2020

January 30, 2021

\begin{abstract}
Background: Covid-19 diagnosis may be difficult in patients with pleural effusion and pneumonia symptoms with heart failure and having atypical clinical course with consecutive negative RT-PCR tests. Case Report: An 80-year-old woman, with the history of chronic dyspnea, previous coronary bypass surgery, heart failure, and obesity was diagnosed as Covid-19 with chest tomography findings. Conclusion: It is observed that despite the improvement of symptoms in the first week, it is possible for a second phase to develop in such patients, which may result in mortality so clinicians should keep in mind that negative consecutive RT-PCR tests do not necessarily rule out Covid-19 pneumonia with bilaterally pleural effusion.
\end{abstract}

Keywords: Computed Tomography, Dyspnea, Heart Failure, Pleural Effusion, Polymerase Chain Reaction.

\section{Introduction}

The diagnosis of Covid-19 can be established on the basis of a suggestive clinical history and the detection of SARS-Cov-2 RNA in respiratory secretions [1]. Chest tomography must be performed and commonly shows bilateral groundglass opacities. In this report, the authors wanted to draw attention to the difficulty of diagnosing Covid 19 in elderly patients who have a biphasic clinical course and bilateral pleural effusion at the time of the diagnosis with a medical history of multiple predisposing factors such as diabetes, obesity and chronic heart failure.

\section{Case Report}

An 80-year-old female patient with the history of progressive shortness of breath for 10-days was applied to our outpatient clinic. The patient, who had been in quarantine at home for the last two months, was reported to have attended a private hospital 15 days ago due to upper extremity trauma.
It was informed that she underwent coronary bypass surgery, and was having a history of heart failure, and obesity.

In auscultation, breathing sounds were absent in basal portions of the lungs, bilaterally. Moreover, breathing sounds were rough in the upper and middle zones. Laboratory studies showed a normal leukocyte and decreased lymphocytes count, slightly elevated C-reactive protein and markedly elevated procalcitonin (7.17 ng/mL). Covid-19 RT-PCR test obtained from pharyngeal swab was negative. Cardiomegaly, bilateral pleural effusion, focal ground-glass opacity and patchy consolidation were present in the first chest tomography [Fig.1a,b].

The patient received treatment for heart failure and immunomodulatory therapy for possible Covid-19 and bacterial pneumonia. This treatment led the patient to an improvement in shortness of breath and dry cough. Covid-19 RT-PCR test was 
repeated by another pharyngeal sample and the result was negative once again on the $5^{\text {th }}$ day. The oxygen saturation was normal until the $5^{\text {th }}$ day of hospitalization but on the $7^{\text {th }}$ day, somehow, the patient's oxygen saturation in arterial blood gas test decreased to $85-90 \%$ range. A chest tomography was performed and a new pharyngeal sample was taken just before and after transfer to the intensive care unit. Treatment with high-dose favipiravir regimen, $1200 \mathrm{mg}$ favipavir twice daily for 1 day followed by $800 \mathrm{mg}$ favipiravir twice daily for 4 days was started. Despite application of CPAP (continuous positive airway pressure), the patient had to be intubated when the oxygen saturation in arterial blood gas test decreased to $75 \%$. On the $9^{\text {th }}$ day, RT-PCR test was positive. On the $11^{\text {th }}$ day, unfortunately, cardiac arrest occurred after convalescent plasma therapy and the patient was lost.

\section{Discussion}

Fever, dry cough and shortness of breath are the most common symptoms in Covid-19 infection. Throat, head and diffuse muscle aches, diarrhea and vomiting are less common [2]. In accordance with this information, our patient had mild fever $\left(37.6^{\circ} \mathrm{C}\right)$, dry cough and shortness of breath, at the first physical examination. White blood cell count was normal but mild lymphopenia was also present at the initial laboratory tests. On the other hand, prothrombin time, ferritin and D-dimer levels were normal. Procalcitonin was apparently high. In many publications, it has been reported that in severe cases of Covid-19, lymphopenia develops and that acute inflammatory markers, such as ferritin and D-dimer, increase $[3,4]$. The obviously observed high procalcitonin level has suggested a bacterial pneumonia [4].

The results of nasopharyngeal swabs on the $1^{\text {st }}$ and $5^{\text {th }}$ days were both negative. Samples of virus-specific IgG and IgM antibodies, which were taken at the beginning of the disease, were also negative. However, the nasopharyngeal swab test
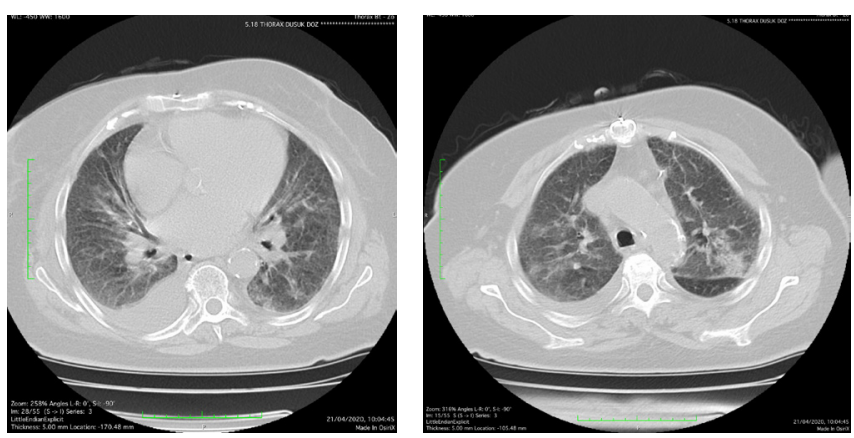

Fig.1: Chest CT scan obtained at admission shows (a) Focal ground-glass opacity associated with inter-lobular and intra-lobular septal thickening in the lower lobes and bilateral pleural effusions and cardiomegaly; (b) Groundglass opacities associated with interlobular septal thickening in the upper lobes and patchy consolidation in the left upper lung.

result was found to be positive on the $9^{\text {th }}$ day when the patient developed hypoxemia. The patient was quarantined with pre-diagnosis of Covid-19 infection, heart failure and pneumonia. Based on treatment plan in Turkey, immunomodulation drugs (chloroquine $200 \mathrm{mg}$ twice daily and azithromycin $500 \mathrm{mg}$ per day) was used for possible Covid-19 infection [5]. A broad-spectrum antibiotic (piperacillin/tazobactam $4 \mathrm{~g} / 0.5 \mathrm{~g}$, three times a day) was delivered for possible bacterial pneumonia and treatment for heart failure was added initially. At the end of the $5^{\text {th }}$ day, the patient's complaints decreased significantly however, on the $7^{\text {th }}$ day; fever, cough and shortness of breath got worse. Moreover, oxygen saturation in arterial blood gas test decreased to $85-90 \%$ range. The result of the nasopharyngeal swab sample taken at this time was positive. Small number of such cases with negative consecutive RT-PCR results have been reported in the literature [6,7]. The RTPCR test performed with nasopharyngeal swab sample is the most important criteria in Covid-19 pneumonia. It has been reported that virus can be identified in blood, body fluids, oropharyngeal and nasopharyngeal secretions [8,9]. False positive results from one time testing was as high as 30$60 \%$ in real Covid- 19 cases $[9,10]$. This high falsenegative rate is the major drawback of this test. 
Insufficient sampling and inefficient virus detection kit (e.g. in transport time) may cause false-negative results. Our patient consecutively had negative test results until the symptoms worsened. It is expected that taking the sputum sample at the time when the infection progresses to the lower respiratory tract or obtaining nasotracheal aspirate or bronchoalveolar lavage under sedation will increase the accuracy rate of the RT-PCR test $[10,11]$. Some authors have emphasized that the disease has become more severe especially after the $5^{\text {th }}$ day $[12,13]$. However, in our case, while the clinical course improved obviously, re-exacerbation of the disease suggested a biphasic course. The lung X-ray (CXR) generally shows bilateral infiltrations but may be normal in the early phase of the disease. The thin slice chest CT imaging is more sensitive and specific and gives important information about the extent of the disease [14].

The appearance of sub-pleural ground glass opacity is considered as the most fundamental finding. Shi et al. have reported this finding as $65 \%$ in 81 Covid-19 patients. They have also reported other findings as follows: interlobular pleural thickening as 35\%, air bronchogram as $47 \%$ and pleural thickening as $32 \%$. In their study, however, the incidence of pleural effusion was only 5\% [15]. Also, chronic heart failure may produce similar appearances on CT [16]. In patient's first chest tomography, there were infiltration with interlobular septal thickening in both lungs, focal ground-glass opacity and pleural effusion [Fig.1a,b]. On the tomography taken on the $7^{\text {th }}$ day, however, there were multiple ground-glass opacities; but this time with more consolidation and interlobular septal thickening and pleural effusion has remained the same [Fig.2a,b].

\section{Conclusion}

The COVID-19 pandemic has presented considerable challenges to clinicians in terms of diagnosis and treatment. Difficulties in diagnosis and changes in the course of the disease make
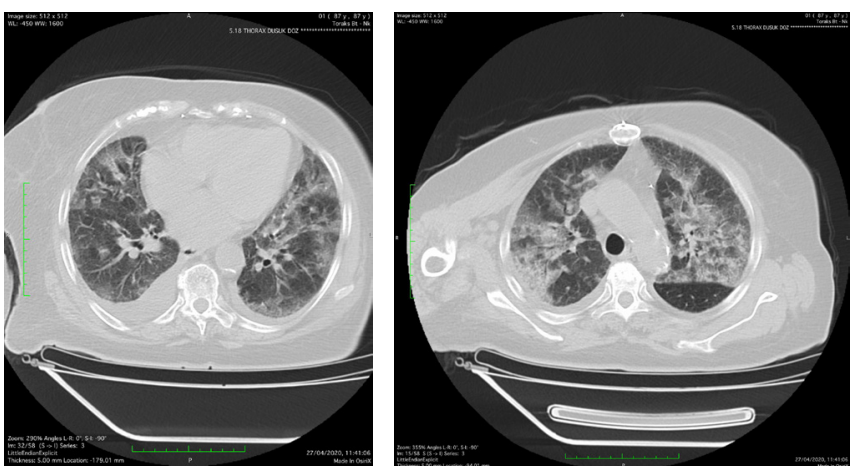

Fig.2: Chest CT scan obtained 6 days after admission (a) The lesions had increased in extent and patchy groundglass opacities affected the bilateral, sub-pleural lung parenchyma in the lower lobes with reduced pleural effusions; (b) The lesions had increased in extent with multiple groundglass opacity with interlobular septal thickening in both upper lobes and more consolidation in the left upper lung.

it difficult to establish a clear approach in the diagnosis and treatment of this disease. Despite improvement in the symptoms of the disease within the first week, the development of acute respiratory failure resulted in the death of our patient. This result may indicate that Covid-19 infection is biphasic. While pleural effusion can be observed frequently in patients with heart failure, it's rare in Covid-19 infection which makes differential diagnosis difficult in these two conditions. More consideration and emphasis should be given to radiological findings, since the consecutive negative RF-PCR test results may delay diagnosis of the Covid-19.

Contributors: SC: manuscript writing and literature review; ND: critical inputs into the manuscript and treatment; NE: literature review and discussion. YB: crtically revised the content for important intellectual contents. SC will act as a study guarantor. All authors approved the final version of this manuscript and are responsible for all aspects of the study. Funding: None; Competing interests: None stated.

\section{References}

1. Coronavirus disease 2019 (COVID-2019). Centers for disease control and prevention. Available at https:// www.cdc.gov/mmwr/Novel_Coronavirus_Reports.html. Accessed May 18, 2020. 
2. Jiang F, Deng L, Zhang L, Cai Y, Cheung CW, Xia Z. Review of the clinical characteristics of coronavirus disease 2019 (Covid-19). J Gen Intern Med. 2020;35: 1545-1549.

3. Abbasi-Oshaghi E, Mirzaei F, Farahani F, Khodadadi I, Tayebinia H. Diagnosis and treatment of coronavirus disease 2019 (COVID-19): Laboratory, PCR and chest CT imaging findings. Int J Surg. 2020;79:143-153.

4. Meisner M. Procalcitonin (PCT) - A new, innovative infection parameter. Biochemical and clinical aspects, New York: Berlin Brahms - Diagnostica; 1996.

5. Official Covid 19 treatment procedure in Turkey: Available at https://apps.who.int/iris/bitstream/ handle/10665/335803/WHO-EURO-2020-1168-4091455408-eng.pdf. Accessed: June 4, 2020.

6. Li H, Feng J, Chen L. Multiple negative of testing of covid-19 pneumonia: A case report. Research Square, 2020. Available at https://www.researchsquare.com/ article/rs-16094/v1. Accessed: June 4, 2020.

7. Zhao X, Liu B, Yu Y, Wang X, Du Y, Wu X. Covid-19 patient with initially negative RT-PCR. Eurorad, 2020. Available at https://www.eurorad.org/case/16631. Accessed: June 4, 2020.

8. Feng H, Liu Y, Lv M, Zhong J. A case report of COVID-19 with false negative RT-PCR test: necessity of chest CT. Jpn J Radiol. 2020;28:409-410.

9. Yang Y, Yang M, Shen C, Wang F, Yuan J, Li J, et al. Evaluating the accuracy of different respiratory specimens in the laboratory diagnosis and monitoring the viral shedding of 2019-nCoV infections. MedRxiv 2020. Available at https://www.medrxiv.org/content/10 .1101/2020.02.11.20021493v2. Accessed: June 4, 2020.

10. Ai T, Yang Z, Hou H, Chenao Zhan, Chong Chen, Wenzhi Lv, et al. Correlation of chest CT and RT-PCR testing in coronavirus disease 2019 (COVID-19) in China: a report of 1014 cases. Radiology. 2020; 296(2):E32-E40.

11. Wang Y, Kang H, Liu X, Tong Z. Combination of RTqPCR testing and clinical features for diagnosis of Covid-19 facilitates management of SARS-CoV-2 outbreak. J Med Virology Commentary. 2020;9:538539.

12. Shi H, Han $\mathrm{X}$, Jiang $\mathrm{N}$, Cao $\mathrm{Y}$, Alwalid $\mathrm{O}, \mathrm{Gu}$ J, et al. Radiological findings from 81 patients with COVID-19 pneumonia in Wuhan, China: a descriptive study. Lancet Infect Dis. 2020;20:425-434.

13. Zhou F, Yu T, Du R, Fan G, Liu Y, Liu Z, et al. Clinical course and risk factors for mortality of adult inpatients with COVID-19 in Wuhan, China: a retrospective cohort study. Lancet. 2020;395:1054-1062.

14. Bao C, Liu X, Zhang H, Li Y, Liu J. COVID-19 Computed Tomography findings: a systematic review and meta-analysis. J Am Coll Radiol. 2020;17(6):701709.

15. Ye Z, Zhang Y, Wang Y, Huang Z, Song B. Chest CT manifestations of new coronavirus disease 2019 (COVID-19): a pictorial review. Eur Radiol. 2020;30(8):4381-4389. 